\title{
ESTUDIO ESTADÍSTICO-DESCRIPTIVO SOBRE LOS CONTENIDOS Y LÍNEAS DE INVESTIGACIÓN DEL ANUARIO MUSICAL (vol. 1, 1946 al vol. 51, 1996)
}

\author{
Equipo de trabajo dirigido por \\ Mariano LAMBEA \\ y formado por \\ Javier DUQUE GUTIÉRREZ \\ Víctor ESBERT TOBEÑA \\ Xavier GÓMEZ SÁEZ \\ Francesc QUESADA SOLÀ
}

\begin{abstract}
This article offers the diachronic panorama on the contents, methodology and research lines of the musicological periodical Anuario Musical, from volume 1 (1946) to volume 51 (1996). Scientific contents of the articles are clasified according to the following seven thematical areas: "National Musicology", "International Musicology", "National Ethnomusicology", "International Ethnomusicology", "Organology", "Biographies" and "Sources and Documentation". An authors' index ordered alphabetically is also given, including number of collaborations for every author, the cast of the different editorial boards of the periodical along its history, a short description of the period covered by every editorial board, and a brief note on the more representative authors. Descriptive graphics are also provided.
\end{abstract}

\section{Resumen}

Se ofrece una panorámica diacrónica sobre los contenidos, metodología y líneas de investigación de la revista musicológica Anuario Musical, desde el volumen 1 (1946) hasta el volumen 51 (1996). Los contenidos científicos de los artículos se clasifican en las siete áreas temáticas siguientes: «Musicología nacional», «Musicología internacional», «Etnomusicología nacional», «Etnomusicología internacional», «Organología», «Biografías» y «Fuentes y documentación». Se ofrece un listado alfabético de autores con su número de artículos, los diversos consejos editoriales de la revista, la descripción de cada período de dirección y una breve reseña de los autores que han aportado un mayor número de colaboraciones. Se incluyen gráficos de carácter descriptivo.

\section{Introducción}

El objetivo del presente trabajo es facilitar una serie de datos sobre el contenido y la trayectoria científica de la revista musicológica Anuario Musical, desde el volumen 1 (1946) hasta 
el volumen 51 (1996). No se trata de trazar la historia de esta revista, aunque a lo largo del trabajo puedan aparecer algunas referencias sobre ese aspecto, sino de elaborar un informe lo más detallado posible sobre las actividades de dicha revista, con vistas a situar y valorar su evolución científica en el contexto de la musicología española de la segunda mitad de nuestro siglo. Se trata, en definitiva, de conocer a fondo las aportaciones realizadas hasta el momento presente y saber con certeza qué aspectos y prioridades de la investigación se han cubierto.

Conviene anticipar que con la radiografía que vamos a practicar en el Anuario Musical, no tendremos una visión de conjunto sobre el desarrollo y evolución de nuestra disciplina en esta segunda mitad de siglo, ya que faltarán los datos de otras revistas como son Revista de Musicología (1978) ${ }^{1}$, Recerca Musicològica $(1981)^{2}$, Cuadernos de Sección. Música (1983) ${ }^{3}$, Nassarre $(1985)^{4}$, Música. Revista del Real Conservatorio Superior de Música (1994) ${ }^{5}$, Quodlibet. Revista de Especialización Musical (1995) ${ }^{6}$ y Cuadernos de Música Iberoamericana (1996) 7 que, aunque no tan veteranas como el Anuario Musical $(1946)^{8}$, no por ello han dejado de efectuar una interesante y muy útil aportación a la ciencia musicológica hispánica. Sin embargo, estos datos que faltan pueden cubrirse rápidamente por quien quiera sumarse a este tipo de trabajo. Sí obtendremos, en cambio, datos fiables sobre la producción científica evaluada en artículos que se ha llevado a término en el antiguo Instituto Español de Musicología del CSIC, hoy Departamento de Musicología de la Institución «Milá y Fontanals» del CSIC.

Por otra parte, parece claro que la labor titánica, y heroica en muchos casos, de un solo investigador va a ceder paso ahora a una labor conjunta perfilada en equipos de investigación y desarrollada en el seno de instituciones y departamentos con intereses y objetivos comunes, y que mantengan relaciones afines de contenido y metodología. Éste ha sido precisamente el fruto de nuestro trabajo: una labor de equipo que se ha gestado durante varios meses, y cuyo resultado se pone ahora a disposición de cuantos necesiten esta información.

\section{Descripción de los conceptos empleados}

Se han definido una serie de conceptos que en conjunto forman la herramienta o medio que se ha utilizado para obtener los datos. Se han establecido los siguientes tres grandes bloques en los que se ha centrado la búsqueda:
A) Identificación
B) Consejos editorales
C) Temática de los artículos

1. Sociedad Española de Musicología.

2. Institut de Documentació i d'Investigació Musicològiques «Josep Ricart i Matas» (Universitat Autònoma de Barcelona).

3. Sociedad de Estudios Vascos.

4. Sección de Música Antigua de la Institución «Fernando el Católico» (Excma. Diputación Provincial de Zaragoza).

5. Real Conservatorio Superior de Música de Madrid.

6. Aula de Música (Universidad de Alcalá de Henares).

7. Instituto Complutense de Ciencias Musicales (Sociedad General de Autores y Editores).

8. Departamento de Musicología de la Institución «Milá y Fontanals» (Consejo Superior de Investigaciones Científicas). 
ESTUDIO ESTADÍSTICO-DESCRIPTIVO SOBRE LOS CONTENIDOS Y LÍNEAS DE INVESTIGACIÓN DEL ANUARIO MUSICAL

\section{A) Identificación}

Equivale al DNI de la revista, ya que son los datos que la particularizan y la hacen única e irrepetible. Dentro de este bloque podemos distinguir:

Título. Aquella palabra o conjunto de palabras que dan nombre a la revista y que se utiliza para referirse a ella.

Subtítulo. Aquella palabra o conjunto de palabras que complementan al título, ampliando o concretando su significado, pero siempre con un sentido de aclaración del título.

Periodicidad. Volúmenes y números. Aquí nos referimos al tiempo transcurrido entre la edición de los diferentes volúmenes y números de la revista, especificando los casos excepcionales de volúmenes dobles o triples.

País. Especificación del país de edición de la revista.

Lugar de edición. Especificación de la ciudad o población donde se edita la revista.

Institución. Especificación del/de los organismo/s que realizan la edición de la revista y de sus cambios de denominación a lo largo del tiempo.

Número aproximado de páginas. Especificación del número medio de páginas por volumen así como de los volúmenes que se desvían de esa media.

\section{B) Consejos editoriales}

Aquí se consignan los diferentes grupos de personas que se han ocupado de la confección de la revista y cuya función ha sido, entre otras, la selección de artículos, la búsqueda de colaboraciones, etc. Con ello se ha dado unidad y coherencia de criterio a la publicación, de forma que ésta adquiera una personalidad y perfil científico concretos. Se especifican los siguientes cargos, con el nombre y apellidos de la persona que los ocupa:

- Director

- Subdirector

- Secretario

- Vocal

\section{C) Dedicatorias}

Se consignan aquí los volúmenes que han sido dedicados a compositores, musicólogos, a la conmemoración de anivesarios e In memoriam.

D) Autores

Listado, por orden alfabético, de autores nacionales e internacionales que han escrito en el Anuario Musical. Los nombres de los autores aparecen normalizados para su mejor consulta. Asímismo se especifican los artículos en colaboración. 


\section{E) Temática de los artículos}

Se ha intentado hacer una clasificación de los temas de todos los artículos publicados en la revista y en todos sus volúmenes. No ha sido tarea fácil, ya que algunos artículos se pueden considerar como pertenencientes a más de un apartado; en estos casos se ha dado más importancia a la temática global del artículo en el momento de decidir a qué grupo de materia pertenece. En cualquier caso la regla adoptada ha sido la de asignar a cada artículo un único grupo temático.

Los grupos temáticos —o de materias - establecidos son los siguientes:

- Musicología histórica. Recoge los artícuos de investigación musicológica que, tal como la misma ciencia musicológica divide sus áreas, tienen un enfoque específicamente histórico. Se incluyen por tanto aquí los estudios de la música culta occidental desde los tiempos más remotos hasta nuestros días. Hemos distinguido en este grupo temático dos apartados:

- Musicología histórica nacional. Si el objeto de estudio pertenece o afecta a una, a varias o a la totalidad de las comunidades españolas.

- Musicología histórica internacional. Si el objeto de estudio pertenece o afecta a uno o varios países extranjeros.

- Etnomusicología. Aquí se sitúan los artículos de investigación etnomusicológica que, aún haciendo referencia a diferentes temas y que por tanto podrían ser incluidos en ellos, tienen como principal discurso tanto la música popular y de tradición oral como las relaciones de dicha música con el contexto sociocultural que la envuele. También hemos distinguido aquí dos apartados que buscan la misma especificación que la división realiza en el tema de Musicología histórica:

- Etnomusicología nacional.

- Etnomusicología internacional.

- Organología. Se recogen aquí los artículos cuyo tema principal se refiere a uno o varios instrumentos musicales, incluyendo su construcción, historia, mecanismos o cualquier otro aspecto.

- Biografías. Se trata de un grupo temático dedicado a la vida y obra de personas cuya actividad posee relevancia histórica o actual.

- Fuentes y documentación. Aquí se incluyen artículos que recogen datos o información sobre cualquier tema musicológico y que constituyen por tanto el primer paso de la investigación.

\section{Obtención de datos y metodología}

Una vez definida la herramienta de trabajo se ha procedido a su aplicación y posterior anotación de datos directos que, una vez orientados según la perspectiva del estudio, han pasado a ser datos susceptibles de ser interpretados.

La metodología empleada ha sido:

- La consulta y vaciado de cada volumen del Anuario Musical.

- La puesta en común, después de discusión, de la pertenencia y asignación del grupo temático correspondiente a cada artículo?.

9. En este momento la aportación del profesor Mariano Lambea fue decisiva para la marcha del trabajo. 
- La informatización de los datos, introduciéndolos en las aplicaciones informáticas adecuadas. En concreto se ha trabajado con una base de datos, con una hoja de cálculo y con un programa de dibujo para los gráficos más elaborados.

- El uso de las aplicaciones informáticas en función de la línea de trabajo del estudio, con lo que se han obtenido los datos finales.

Los datos obtenidos se han agrupado en los apartados que se detallan a continuación.

\section{Datos de identificación del Anuario Musical}

$\begin{array}{ll}\text { Título: } & \text { Anuario Musical } \\ \text { Subtítulo: } & \text { Revista de Musicología del CSIC } \\ \text { Periodicidad: } & \text { Anual, excepto en los siguientes años y volúmenes: 1973/74 (28- } \\ & \text { 29); 1976/77 (31-32); 1978/80 (33-35) y 1984/85 (39-40). } \\ \text { País: } & \text { España } \\ \text { Lugar de edición: } & \text { Barcelona } \\ \text { Institución editorial: } & \text { Consejo Superior de Investigaciones Científicas } \\ & \text { - Instituto Español de Musicología: vol. 1 (1946) al vol. 39-40 } \\ & \text { (1984/85). } \\ & \text { - U.E.I. [Unidad Estructural de Investigación] de Musicología de la } \\ & \text { Institución «Milá y Fontanals»: vol. 41 (1986) al vol. 48 (1993). } \\ & \text { - Departamento de Musicología de la Institución «Milá y Fonta- } \\ & \text { nals»: desde el vol. } 49 \text { (1984) en adelante. } \\ & \text { Entre 250 y 300 páginas por volumen, excepto el vol. 36 (1981) y } \\ & \text { el vol. } 37 \text { (1982), que tienen unas 170 páginas, respectivamente, y } \\ & \text { el vol. } 45 \text { (1990) con } 350 \text { páginas. }\end{array}$

\section{Consejos editoriales del Anuario Musical}

El Anuario Musical fue fundado por Higinio Anglés Pamies en 1946. Desde entonces, los Consejos editoriales que se han sucedido y que constan impresos en los diferentes volúmenes de la revista, han sido los siguientes:

- Primer Consejo editorial: vol. 25 (1970)

Director Miguel Querol Gavaldá

Secretario José $\mathrm{M}^{\mathrm{a}}$ Llorens Cisteró

- Segundo Consejo editorial: del vol. 26 (1971) al vol. 33-35 (1978/80)

Director Miguel Querol Gavaldá

- Tercer Consejo editorial: del vol. 36 (1981) al vol. 41 (1986)

Director José $\mathrm{M}^{\mathrm{a}}$ Llorens Cisteró 
- Cuarto Consejo editorial: vol. 42 (1987)

$\begin{array}{ll}\text { Director } & \text { José } \mathrm{M}^{\mathrm{a}} \text { Llorens Cisteró } \\ \text { Secretario } & \text { Mariano Lambea Castro }\end{array}$

- Quinto Consejo editorial: del vol. 43 (1988) al vol. 44 (1989)

Director José V. González Valle

Jefe de Redacción Josep Martí Pérez

Secretario Mariano Lambea Castro

- Sexto Consejo editorial: del vol. 45 (1990) al vol. 48 (1993)

Director José V. González Valle

Subdirector Josep Pavia Simó

Jefe de Redacción Josep Martí Pérez

Secretario Mariano Lambea Castro

- Séptimo Consejo editorial: del vol. 49 (1994) en adelante

Director José V. González Valle

Subdirector Josep Pavia Simó

Jefe de Redacción Josep Martí Pérez

Secretario Mariano Lambea Castro

Vocales Antonio Ezquerro Esteban

Luis A. González Marín

\section{Volúmenes dedicados}

Vol. 8 (1953)

Vol. 17 (1962)

Vol. 18 (1963)

Vol. 19 (1964)

Vol. 21 (1966)

Vol. 27 (1972)

Vol. 36 (1981)

Vol. 37 (1982)

Vol. 39-40 (1984-85) In Memoriam Marius Schneider

Vol. 41 (1986)

Vol. 42 (1987)

Vol. 43 (1988)

Vol. 49 (1994)

Vol. 51 (1996)

Juan Bautista Cabanilles

José Subirá en su 80 aniversario

In Memoriam Felipe Pedrell
IV Centenario de la muerte de Cristóbal de Morales

Mosén Francisco de Paula Baldelló

Antonio de Cabezón en el IV centenario de su muerte

Miguel Querol Gavaldá en su 70 aniversario

Miguel Querol Gavaldá en su 70 aniversario

In Memoriam P. José Antonio de Donostia

José Romeu i Figueres en su 70 aniversario

In Memoriam y Centenario Higinio Anglés

Josep Maria Llorens en su 70 aniversario

Cincuentenario del Anuario Musical (incluye una Ilustración especial con motivo de la entrega del Premio Nacional «Rey Don Juan Carlos I» al Dr. Luis Antonio González Marín 


\section{Los autores del Anuario Musical}

A lo largo de 51 volúmenes, desde 1946 hasta 1996, en el Anuario Musical han participado hasta un total de 107 autores nacionales y 114 autores extranjeros. Los artículos de la revista suman en total 527. A continuación ofrecemos un listado por orden alfabético de los autores, tanto nacionales como internacionales, especificando el número de artículos que han escrito. Los autores precedidos de un asterisco estuvieron o están vinculados al Instituto Español de Musicología o al Departamento de Musicología, respectivamente.

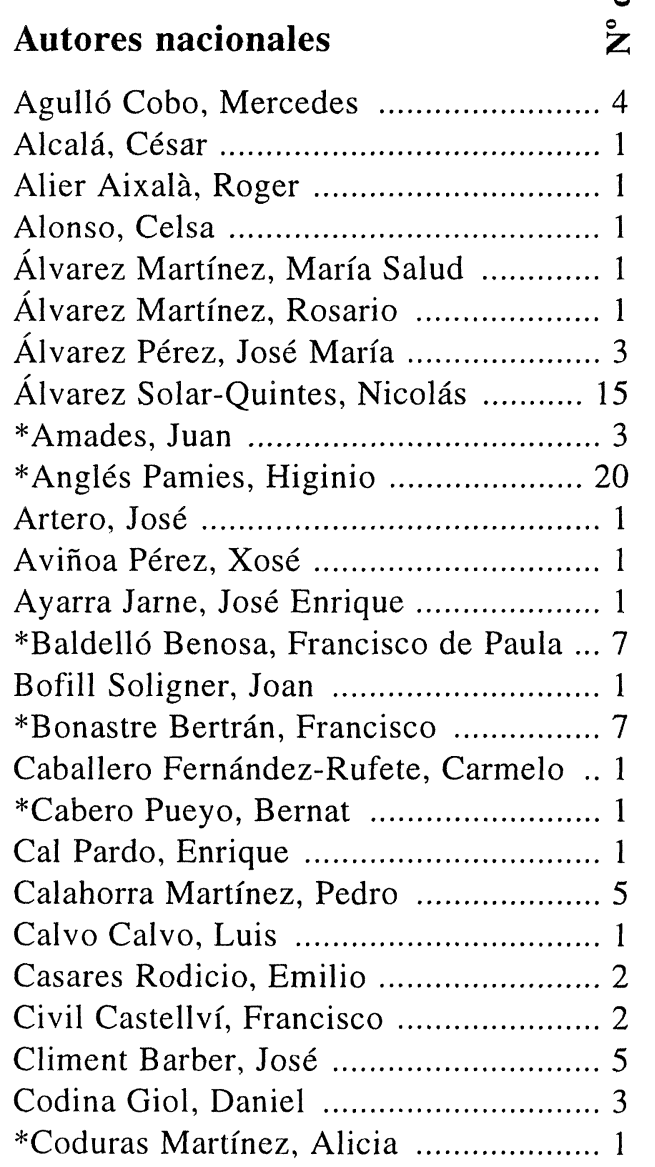

Anuario Musical, 52 (1997)

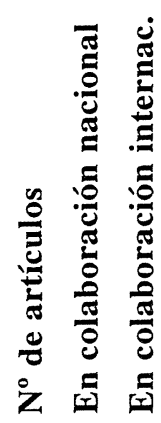

\begin{tabular}{|c|c|c|c|}
\hline & & ortés Mir, Francesc ............................. 1 & \\
\hline & - & Costa, Alejandro & 1 \\
\hline & - & *Costa Vicent, Ramón & - \\
\hline & - & Crespí González, Joana .. & - \\
\hline & - & *Crivillé Bargalló, José ............... & - \\
\hline & - & Díaz, Joaquín & - \\
\hline & - & *Dolcet Rodríguez, Josep & - \\
\hline & - & *Donostia, José Antonio de ................. 6 & 1 \\
\hline & - & Durán Gudiol, Antonio ........................ 1 & - \\
\hline & - & El Molar, Nolasco de ......................... 1 & - \\
\hline & - & Erdozaín, Carmelo ................................. 1 & - \\
\hline & - & *Ester Sala, María Asunción ................... 3 & 3 \\
\hline & - & *Ezquerro Esteban, Antonio .................. 4 & 1 \\
\hline & - & García Chico, Esteban ........................... 2 & - \\
\hline & - & García Fraile, Dámaso .......................... 2 & - \\
\hline & - & *García Matos, Manuel ........................ 8 & - \\
\hline & - & *Gil García, Bonifacio ......................... 2 & - \\
\hline & - & *Gómez Muntané, María del Carmen ... 1 & - \\
\hline & 1 & González Barrionuevo, Herminio .......... 2 & - \\
\hline & - & *González Marín, Luis Antonio ............ 3 & 1 \\
\hline & - & *González Valle, José Vicente .............. 9 & - \\
\hline & - & Gosálvez Lara, Carlos José . ................... 1 & - \\
\hline & - & Gregori Cifré, Josep Maria ……........... 2 & _- \\
\hline & - & Gutiérrez, Carmen Julia ..... & - \\
\hline & - & Hernández Ascunce, Leocadio ............... 2 & - \\
\hline & - & Hoyos Sancho, Nieves de.. & - \\
\hline
\end{tabular}




\begin{tabular}{|c|c|c|c|c|c|}
\hline 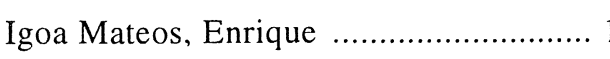 & 1 & - & 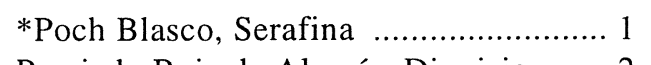 & 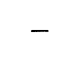 & \\
\hline 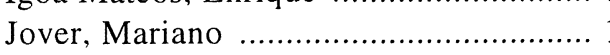 & - & - & Preciado Ruiz de Alegría, Dionisio ....... 2 & - & 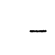 \\
\hline kamaña, José María ............................... & - & - & Pujol, David & - & 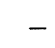 \\
\hline *Lambea Castro, Mariano ....................... & - & - & Pujol, Francisco & - & 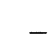 \\
\hline *Larrea, Arcadio de ............................... & - & - & 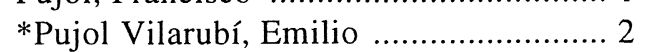 & - & \\
\hline Lizarazu De Mesa, María Asunción ....... & - & - & *Querol Gavaldá, Miguel ..................... 23 & - & \\
\hline 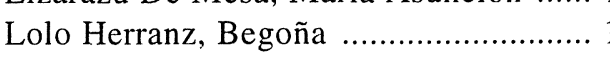 & - & - & Ramos López, Pilar .............................. 1 & - & \\
\hline López Calo, José ......... & - & - & Rifé Santaló, Jordi ...................... & - & \\
\hline López Martínez, Nicolás ... & - & - & Robledo Estaire, Luis .............................. 1 & - & \\
\hline 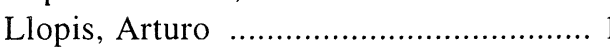 & - & - & *Romeu Figueras, José & - & \\
\hline Llordén, Andrés ................................... 10 & - & - & Rossell, Antoni ............ & - & - \\
\hline *Llorens Cisteró, Jose María ................ 27 & 1 & - & Rubio Calzón, Samuel ........ & - & - \\
\hline Madurell Marimón, José María .............. & 1 & - & Sagasta Galdós, Julián ............................ 1 & - & - \\
\hline *Martí Pérez, Josep .................... & - & - & *Salazar, Adolfo .......... & - & - \\
\hline *Martín Moreno, & - & - & Sancho Campo, Ángel. & - & - \\
\hline Medina Álvarez, Ánge & - & - & Sanz, Clemente .............. & - & - \\
\hline Manuel & - & - & Sevillano, Justo . & - & - \\
\hline$* \mathrm{Mo}$ & - & - & Siemens Hernández, Lothar & - & - \\
\hline Olart & - & - & Carmen & - & - \\
\hline Otao & - & - & Sob & - & - \\
\hline cio ................... & - & - & ña Ibáñez, H & - & - \\
\hline (n............... & - & - & $\ldots \ldots 1$ & - & - \\
\hline 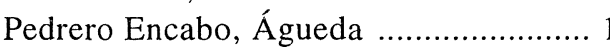 & - & - & .... 16 & - & - \\
\hline & - & - & ..... 3 & 1 & \\
\hline 1 & 1 & - & $\mathrm{Ur}$ & - & \\
\hline 2 & - & - & Vilar Torrens, Jos & 3 & \\
\hline 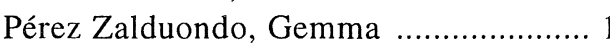 & - & - & & & \\
\hline & - & - & & & \\
\hline Plaza Navas, Miguel Ángel & - & - & Tota & 14 & 1 \\
\hline utore & 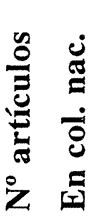 & 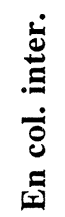 & Autores internacionales & 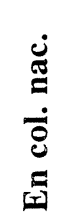 & 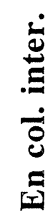 \\
\hline 1 & 1 & - & Bo & - & \\
\hline & 2 & - & $\mathrm{Br}$ & - & \\
\hline Ari & - & - & $\mathrm{Br}$ & - & - \\
\hline Ave & 2 & - & 1 & - & - \\
\hline & - & - & & - & - \\
\hline & - & - & $\ldots 1$ & - & \\
\hline & - & - & $\ldots 1$ & - & \\
\hline & - & - & Cas & - & \\
\hline & 2 & - & $\mathrm{Ca}$ & - & \\
\hline & 1 & - & Claro Valdés, S & - & \\
\hline Bol & 1 & - & & - & \\
\hline Bourligueux, Guy & 1 & - & Corrêa de Azevedo, Luiz Heitor & - & \\
\hline
\end{tabular}




\begin{tabular}{|c|c|c|c|c|c|}
\hline 1 & & - & *Müller Lancé, Karl Heinz .................... 3 & & \\
\hline etko, Dragontin ..... & & - & Mutatkar, Sumati ….............................. 1 & - & - \\
\hline Chailley, Jacques ....... & - & - & Navarrini, Lucia . & 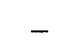 & 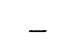 \\
\hline Chaudhary, Subhadra ............................. 1 & - & - & Nelson, Bernadette & - & \\
\hline Devoto, Daniel ..................................... 1 & - & - & *Pelinski, Ramón Adolfo & & \\
\hline Doderer, Gerhard & - & - & Piccus, Jules ......................... & - & \\
\hline Eggebrecht, Hans Heinrich & $r$ & - & Pietzsch, Gerhard & & \\
\hline El Mahdi, Salah ................................ 1 & & - & Powell Jr., Linton E. . & & - \\
\hline mer, Ernest .............................. 1 & & 1 & Quang Hai, Tran .................................. 1 & - & - \\
\hline (n) & - & 1 & Raley, Michael J. ............................... 1 & - & - \\
\hline Ferrari Barassi, Elena & - & - & Rault Leyrat, Lucie ................ & & - \\
\hline Fischer, Kurt von ........ & - & - & Ree Bernad, Nelly van ........... & - & - \\
\hline Frotscher, Gotlhold ................................ 1 & - & - & Reimann, Margarete ..... & - & \\
\hline Gerber, Rudolf von & - & - & Rive, Thomas N. ................................. 2 & - & - \\
\hline Ghis & - & - & , Miriam & - & - \\
\hline Golc & - & - & $\mathrm{Ru}$ & - & - \\
\hline Göll & - & - & Salt & - & - \\
\hline Göll & - & - & San & & - \\
\hline 2 & - & - & 1 & & - \\
\hline & - & - & 11 & - & - \\
\hline 1 & - & - & 1 & & - \\
\hline 1 & - & - & 2 & - & - \\
\hline rald & - & - & $* \mathrm{Sc}$ & - & - \\
\hline Hol & - & - & $\mathrm{Scl}$ & - & - \\
\hline Ho & - & - & $\mathrm{Se}$ & - & - \\
\hline rich & - & - & $\mathrm{Se}$ & - & - \\
\hline Jam & - & - & $\mathrm{Sh}$ & - & - \\
\hline 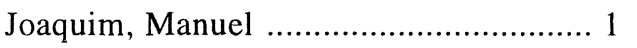 & - & - & $\mathrm{ph}$ & - & - \\
\hline Santiago .................. 19 & - & - & 1 & - & - \\
\hline & - & - & 1 & - & - \\
\hline & - & - & 1 & - & - \\
\hline ............ & - & - & Ste & - & - \\
\hline (. & - & - & bert ........ & - & - \\
\hline ner Leonhard . & - & - & ab, El Fatih El & - & - \\
\hline & - & - & Tib & - & \\
\hline$\ldots \ldots \ldots \ldots \ldots \ldots \ldots \ldots$ & - & - & To & - & \\
\hline ….................. 1 & - & - & 1 & - & \\
\hline . & - & - & 1 & - & - \\
\hline & - & - & $\mathrm{Va}$ & - & \\
\hline 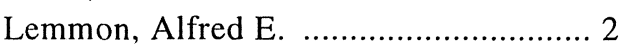 & - & - & Maarten Albert .......................... & - & - \\
\hline 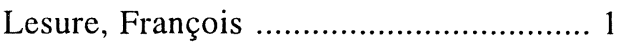 & - & - & 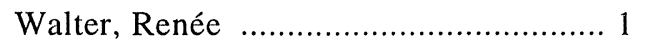 & - & - \\
\hline 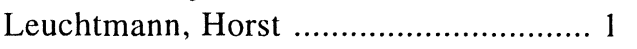 & - & - & Shahak, Susana & - & 1 \\
\hline ................... 1 & - & - & Yin & - & - \\
\hline hammad & - & & Zimmerman, Franck & - & \\
\hline & - & & & & \\
\hline Moody, Ivan ........................................ 1 & - & - & tores: 114 & & 1 \\
\hline
\end{tabular}




\section{Las materias del Anuario Musical}

Todo este volumen de artículos es susceptible de ser clasificado en ámbitos temáticos según su contenido científico. A tal efecto se han establecido siete materias, conceptos o palabras clave que aglutinan los 527 artículos de que consta la revista. Téngase en cuenta que a cada artículo se le ha asignado una única materia, particularmente la que se ha considerado de mayor alcance y significación. En el siguiente cuadro se pueden observar las mencionadas materias, el número de artículos clasificados en ellas y la cantidad de ellos que se deben a autores nacionales y a autores internacionales:

$\begin{array}{lccc}\text { Materias } & N^{o} \text { artículos } & \text { Autores nac. Autores internac. } \\ \text { Musicología nacional } & 155 & 98 & 57 \\ \text { Musicología internacional } & 38 & 92 & 9 \\ \text { Etnomusicología nacional } & 57 & 37 & 20 \\ \text { Etnomusicología internacional } & 34 & 3 & 31 \\ \text { Organología } & 31 & 26 & 5 \\ \text { Biografías } & 45 & 38 & 7 \\ \text { Fuentes y documentación } & 167 & 137 & 30 \\ \text { Totales } & \mathbf{5 2 7} & \mathbf{3 4 8} & \mathbf{1 7 9}\end{array}$

Asímismo, incluimos el siguiente gráfico que representa el «volumen promedio» en cuanto a materias de todos los períodos de publicación del Anuario Musical. Se trata, por consiguiente, de un volumen ideal, pero, a la vez, indicativo de las aportaciones realizadas en la revista.

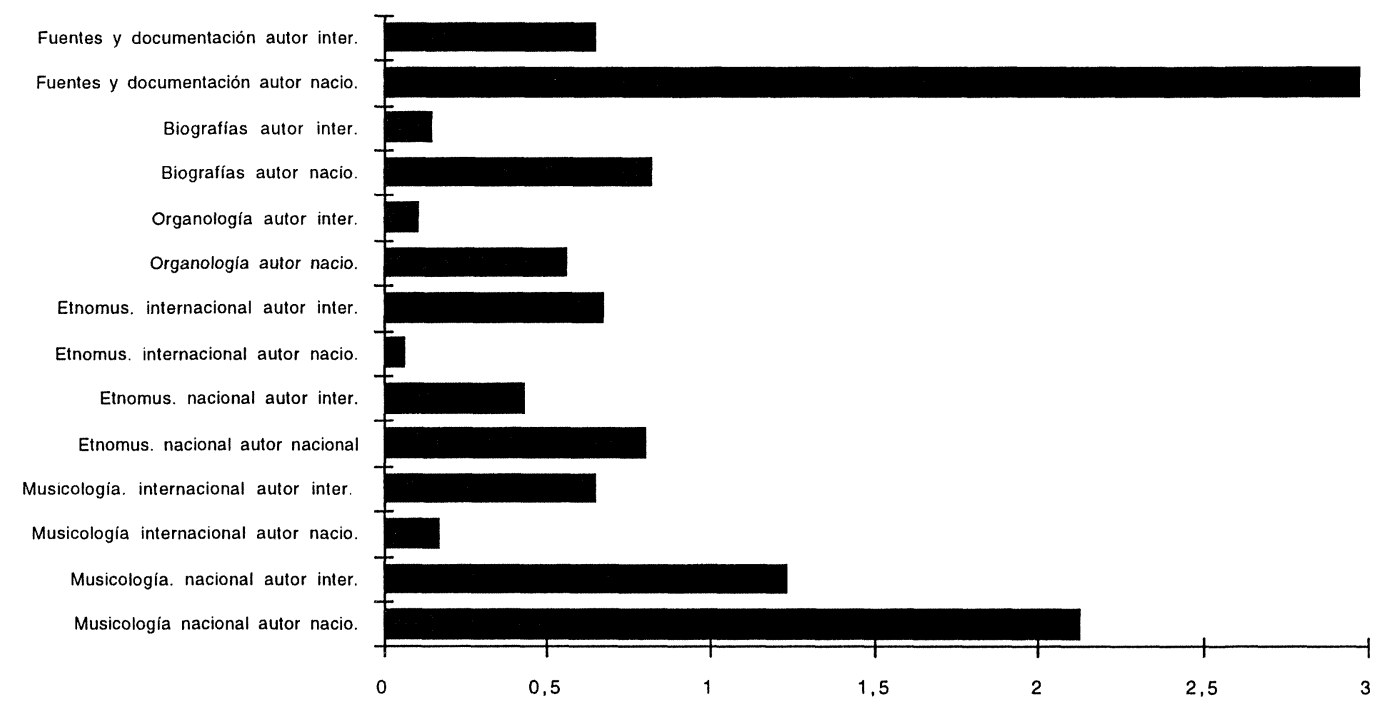




\section{Interpretación de datos}

Partiendo de los datos obtenidos se procede a un cruzamiento entre ellos para darles mayor relieve y sentido; de esta manera pasan a ser datos listos para obtener conclusiones. El equipo de trabajo procedió a ese cruzamiento, que se expone a continuación, pero consideró necesario un estudio más profundo para llegar a la siguiente fase de conclusiones. Es por esto que este trabajo en su conjunto, al faltarle - y en espera de - las conclusiones, se puede considerar claramente como de «Fuentes y documentación». A pesar de ello se han podido vislumbrar ciertas proyecciones de las conclusiones que a manera de sugerencia particular aparecen en esta interpretación de datos que ahora exponemos. Muchas de estas interpretaciones de datos están apoyadas por los gráficos obtenidos con la hoja de cálculo utilizada para analizar los datos.

\section{Relación entre autores y materias}

Existe en todas las materias mucha mayor presencia de artículos referidos a música nacional que a música internacional. De lo que fácilmante se deduce que el Anuario Musical es una revista más ocupada en cuestiones musicales autóctonas que no foráneas.
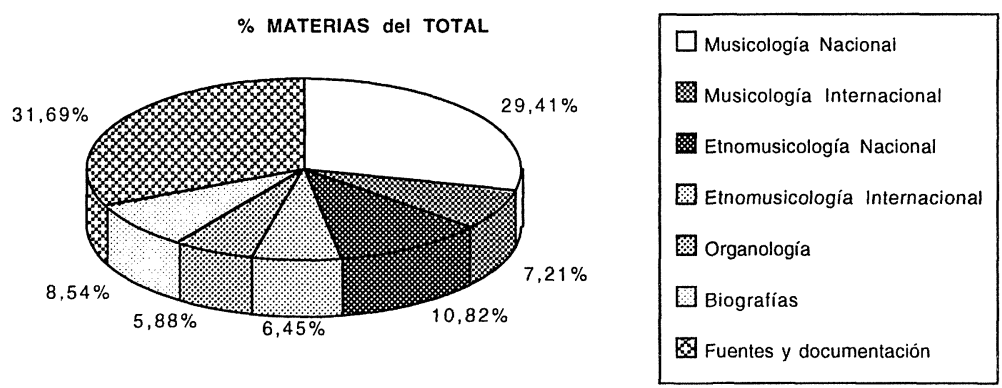

Se dan muchos artículos escritos por autores internacionales, de lo que se desprende una voluntad de enriquecimiento en todos los órdenes a través del contacto con musicólogos de otros países.

Del total de materias, la más tratada por los autores nacionales es «Fuentes y documentación», posiblemente porque la disciplina musicológica en nuestro país ha tenido que desarrollar, en una primera fase de la investigación y con mayor dedicación, la vertiente heurística.

En los artículos de autores internacionales hay mucha menor presencia de estudios sobre «Fuentes y documentación», así como sobre «Organología» y «Biografías», apareciendo mayor número de trabajos analíticos y de carácter reflexivo. Este hecho puede marcar una línea diferenciadora entre la musicología nacional e internacional, aunque hay que tener en cuenta que la 
presencia de los autores internacionales puede parecer sesgada, al ser su colaboración en el Anuario Musical invitada y puntual, y esta circunstancia quizá provoque el condicionamiento de la elección temática desde diversas vertientes. En consecuencia, este tipo de inferencias comparativas no siempre puede resultar correcto.

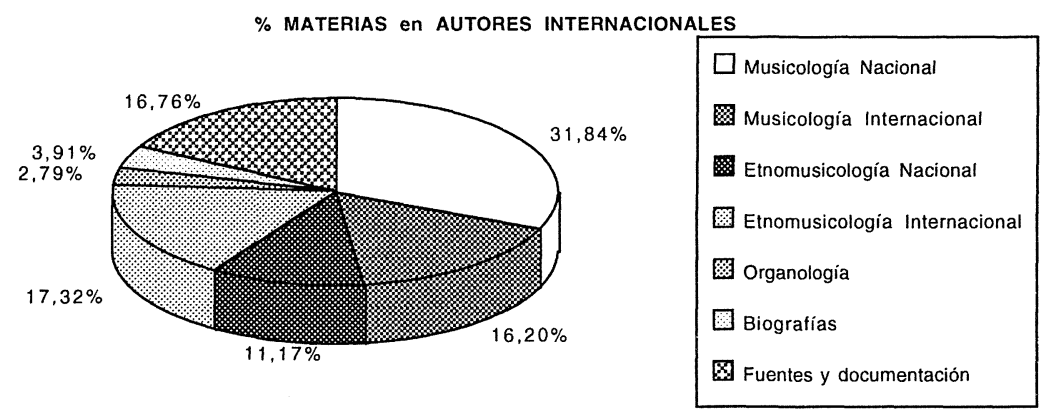

En los autores nacionales se puede observar un menor porcentaje de temas internacionales, ya sean musicológicos o etnomusicológicos. En cambio, los autores internacionales han aportado mayor número de colaboraciones en estos mismos temas respecto a aquéllos. Si comparamos los porcentajes, la proporción de aportaciones en «Etnomusicología internacional» es aproximadamente de 20 a 1 a favor de los autores internacionales, y la proporción en «Musicología internacional» es de algo más de 6 a 1, también a favor de estos últimos autores.
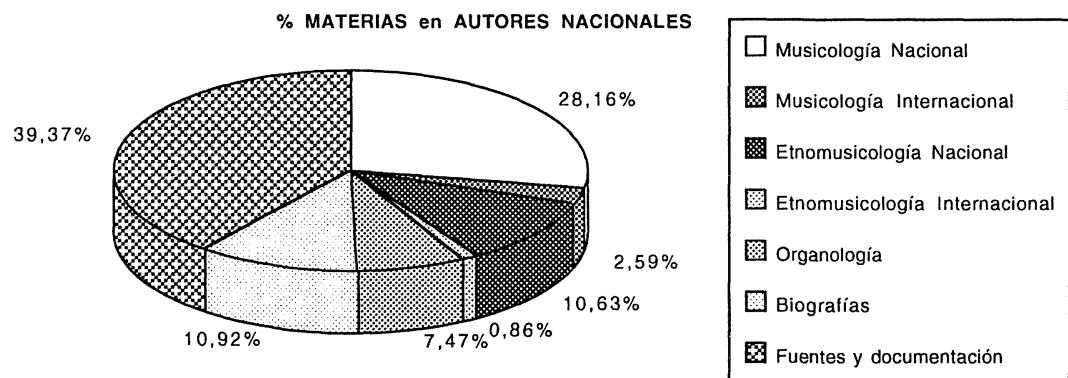

\section{Temáticas y períodos de dirección}

En el período de Higinio Anglés (1946-1969) queda definida la línea metodológica de la revista por la personalidad de su fundador y por las prioridades de la musicología española en aquella época:

- Inicio de colaboraciones y contactos con la musicología internacional, de la cual Anglés era considerado un alto exponente, en una decidida postura de dotar a la musicología española de líneas de investigación modernas y actuales. 
- Monografías de volúmenes dedicados (en la línea de los Festschrifts germanos), y destinados a conmemorar efemérides de músicos españoles importantes con la intención de ahondar en sus obras y biografías y actualizar sus bibliografías.

- Mantenimiento de las proporciones de cada temática durante todo el período, asentando las bases de lo que será hasta hoy la personalidad de la revista, esto es, un especial énfasis en el tema de «Fuentes y documentación», que, a partir de la segunda mitad del período, pasa a tener definitivamente más presencia que «Musicología nacional». Estos dos temas juntos forman el bloque principal del Anuario Musical.

En el período de Miguel Querol (1970-1980) las temáticas inciden, sobre todo, en el bloque principal; el segundo bloque, que está formado por «Etnomusicología nacional» y «Etnomusicología internacional», «Musicología internacional», «Organología» y «Biografías» tienen muy pocas aportaciones de artículos durante el período, exceptuando el de «Biografías», que recibirá un impulso que lo colocará a la cabeza de este segundo grupo de temas. Es de destacar, también, que es en este período cuando se da el número mayor de volúmenes múltiples, o sea, de más de un año. En concreto, dos volúmenes dobles (números 28/29 correspondientes a los años 1973-74 y números 31/32 de los años 1976-77) y uno triple (números 33/34/35 de los años 1978-79-80), que es el último del período. También este período es el de menos volúmenes dedicados: uno, en memoria de Felipe Pedrell.

En el período de José María Llorens (1981-1987), de nuevo, el segundo bloque de temas recibe artículos de forma regular. Los volúmenes dedicados son numerosos y son la causa de aumentos espectaculares en algunas temáticas concretas. Así ocurre en el tema «Etnomusicología internacional», con el volumen In Memoriam dedicado al reconocido etnomusicólogo Marius Schneider (volúmenes 39-40 de los años 1984-85). También con el volumen In Memoriam del Padre Donostia (volumen 41 del año 1986), la «Etnomusicología nacional» vuelve a recibir aportaciones, y esta circunstancia la colocará en una posición favorable para -en la siguiente época, la actual, a cargo de José Vicente González Valle- llegar a liderar el segundo grupo de temáticas, como sucediera en el período de Higinio Anglés, superando al área temática de «Biografías».

El período de José Vicente González Valle (1988-1996) se inaugura con la celebración del centenario del nacimiento de Higinio Anglés (volumen 43 del año 1988). En las cuatro temáticas más numerosas («Fuentes y documentación», «Musicología nacional», «Etnomusicología nacional» y «Biografías») se recuperan las proporciones del período de Anglés, manteniéndose, como en todas las épocas anteriores, el bloque primero en progresión destacada («Fuentes y documentación» y «Musicología nacional»). De nuevo, lo más significativo se encuentra en el grupo segundo; aquí las «Etnomusicologías» reciben un gran impulso que se va sosteniendo; también lo recibe la «Musicología internacional». En cambio, tanto las «Biografías» - que están casi sin aportaciones - como la «Organología», son los temas que apenas tienen movimiento. 
Mariano Lambea, J. Duque, V. Esbert, X. Gómez y F. Quesada

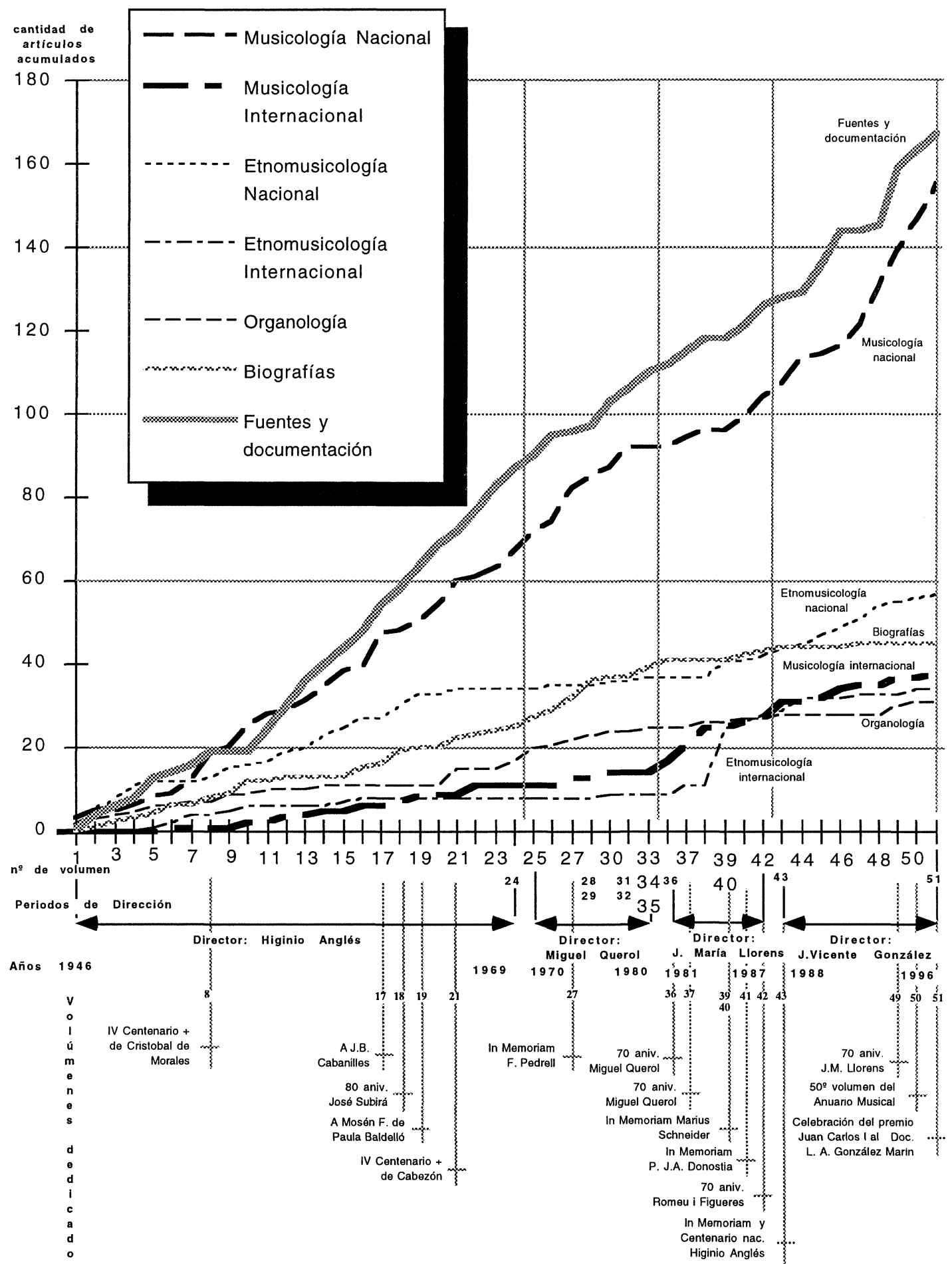




\section{Los autores con más colaboraciones} detalla.

La mayoría de estos autores son nacionales, salvo alguna excepción que a continuación se

El autor con más artículos publicados en la revista es José María Llorens Cisteró con un total de 27 artículos que se distribuyen así:

- 13 sobre «Fuentes y documentación».

- 8 sobre «Biografías».

- 5 sobre «Musicología nacional».

- 1 sobre «Organología».

De estos 27 artículos uno está escrito en colaboración con José María Madurell Marimón. La época de publicación de estos artículos es muy extensa, iniciándose en el año 1953 y finalizando en 1996, pero el período más fértil coincide con su asunción en la dirección de la revista.

El siguiente autor más publicado es Miguel Querol Gavaldá con 23 artículos con la siguiente distribución:

- 13 sobre «Musicología nacional».

- 4 sobre «Fuentes y documentación».

- 3 sobre «Biografías».

- 2 sobre «Etnomusicología nacional».

- 1 sobre «Musicología internacional».

Querol publica estos artículos entre 1947 y 1994, coincidiendo también su etapa más creadora con la época en que se hizo cargo de la dirección del Anuario Musical.

El tercer autor en aportaciones es el fundador de la revista Higinio Anglés Pamies. Es uno de los autores de mayor variedad temática en sus artículos, buena prueba de su amplia curiosidad científica y de su capacidad de dominio sobre los más variados ámbitos de la musicología universal. Tiene 20 artículos publicados, distribuidos de la siguiente manera:

- 9 sobre «Musicología nacional».

- 5 sobre «Fuentes y documentación».

- 2 sobre «Etnomusicología nacional».

- 1 sobre «Etnomusicología internacional».

- 1 sobre «Musicología internacional».

- 1 sobre «Biografías».

- 1 sobre «Organología».

La época de publicación de sus artículos va desde 1947 hasta 1966 y lo hace de forma progresiva y constante.

El siguiente autor en número de artículos publicados es Macario Santiago Kastner, siendo el primero que pertenece al grupo de autores internacionales. Publicó un total de 19 artículos distribuidos así:

- 12 sobre «Musicología nacional».

- 6 sobre «Fuentes y documentación».

- 1 sobre «Musicología internacional». 
Estos artículos fueron publicados entre los años 1946 y 1989, siendo la época más creadora y más constante la comprendida entre 1952 y 1966.

En quinto lugar, pero cuarto en cuanto a autores nacionales, encontramos a José Subirá Puig con 16 artículos de temática muy concentrada:

- 9 sobre «Biografías».

- 7 sobre «Musicología nacional».

La época de publicación de estos artículos es la comprendida entre los años 1946 a 1972, siendo el período más regular el que va de 1948 a 1961.

Los tres autores nacionales siguientes son Nicolás Álvarez Solar-Quintes con 15 artículos, Jaime Moll Roqueta con 12 y José Romeu Figueras con 11.

A continuación viene el segundo autor internacional más publicado en el Anuario Musical; se trata de Marius Schneider con un total de 11 artículos que se distribuyen de la siguiente manera:

- 7 sobre «Etnomusicología nacional».

- 3 sobre «Etnomusicología internacional».

- 1 sobre «Musicología nacional».

Estos artículos son publicados entre 1946 y 1959, y lo son de forma totalmente regular desde su inicio hasta el año 1955.

\section{Epílogo}

Al término de este trabajo creemos que hemos cumplido con nuestro objetivo de tomar el pulso a la actividad científica de la revista Anuario Musical, así como hemos destacado también algunos rasgos específicos de su historia. Hemos facilitado una serie de datos que por sí mismos alcanzan a explicar la realidad de la revista, su evolución y líneas de investigación. En realidad, las conclusiones del trabajo están presentes y detalladas en el contenido de los diferentes epígrafes, pero quizás sea conveniente añadir alguna indicación de cara al futuro, en el sentido de confrontar esta información con otros datos provenientes del resto de revistas de musicología españolas. Será un trabajo a largo plazo que podrá arrojar luz sobre aspectos interesantes del quehacer científico de nuestra disciplina en sus múltiples y diversificadas facetas. 